\title{
Thermalisation of Quantum States
}

\author{
Dorje C. Brody* and Lane P. Hughston ${ }^{\dagger}$ \\ *DAMTP, Silver Street, Cambridge CB3 9EW U.K. \\ $\dagger$ Merrill Lynch International, 25 Ropemaker Street, London EC2Y 9LY U.K. \\ and King's College London, The Strand, London WC2R 2LS, U.K.
}

(September 1, 2018)

An exact stochastic model for the thermalisation of quantum states is proposed. The model has various physically appealing properties. The dynamics are characterised by an underlying Schrödinger evolution, together with a nonlinear term driving the system towards an asymptotic equilibrium state and a stochastic term reflecting fluctuations. There are two free parameters, one of which can be identified with the heat bath temperature, while the other determines the characteristic time scale for thermalisation. Exact expressions are derived for the evolutionary dynamics of the system energy, the system entropy, and the associated density operator.

PACS Numbers : 05.70.Ln, 02.50.Ey, 02.40.Vh, 05.30.Ch

\section{INTRODUCTION}

The construction of a microscopic description for dynamical systems out of equilibrium is a topic of considerable interest in statistical mechanics. While many studies have been pursued in this direction [罒, the subject remains refractory owing to its nonlinear character. In particular, a realistic framework must be simple enough to be tractable, yet sufficiently sophisticated to capture some of the physical essentials. Our goal here is to propose in this spirit an elementary but compelling stochastic model that characterises the thermalisation process for a quantum system in an arbitrary given initial state.

The approach here, though carried out largely within the framework of standard quantum theory, involves a reexamination of the traditional hypotheses forming the basis of quantum statistical mechanics. In particular, we shall take the view that a quantum statistical ensemble, or 'state', is correctly described not simply by a density matrix $\rho_{\beta}^{\alpha}$, but rather by a probability distribution on the space of pure quantum mechanical states. For measurements associated with ordinary quantum mechanical observables this description reduces to the usual one, for which the unconditional expectation of a linear observable is given by the familiar trace formula involving $\rho_{\beta}^{\alpha}$. The advantage of the present formulation, however, is that we can give meaning to the concept of ensembles and other mixed states in nonlinear quantum mechanics [2.33. Furthermore, the fact that the space of pure states has a symplectic structure allows us to introduce the concept of a quantum phase space $\Gamma$, and hence the use of probability distributions on $\Gamma$ as ensembles. In particular, for thermal equilibrium in certain situations we propose the distribution given in (12) below, which we call the canonical $\Gamma$-ensemble. The corresponding density matrix differs in some respects from the conventional density matrix of quantum statistical mechanics, and thus the applicability of the theory may in principle be testable. 
Our first step is to derive the Fokker-Planck equation for the time-dependent probability density function associated with a general Brownian motion with drift on the space of pure quantum states. Since $\Gamma$ is compact, we can apply a theorem of Zeeman to show that there is a special class of drift terms for which, given any initial condition, the resulting asymptotic stationary solution is the canonical $\Gamma$-ensemble described above. The nonequilibrium dynamics we consider here is governed by a nonlinear Schrödinger evolution coupled to a thermal noise term, described by the stochastic differential equation (14). This choice is the simplest possible that leads to thermal equilibrium for arbitrary initial states.

In what follows we use the dynamical law (14) to derive a formula for the total system energy (18), leading to a formula for the heat capacity (19). These ideas are applied, by way of illustration, to the case of a spin one-half particle in a heat bath. Then we derive an expression for the dynamics of the system entropy, given in (24), from which we are able to deduce a fundamental thermodynamic inequality (25). Finally we study the evolution of the density matrix associated with the underlying process. We show that the time development of the density matrix involves a higher moment of the projection operator onto pure states.

\section{QUANTUM HAMILTONIAN DYNAMICS}

We begin by considering the quantum mechanical phase space $C P^{n}$ [2]. This is the space of pure quantum states, given by rays through the origin in the associated Hilbert space. The quantum phase space can be viewed as a $2 n$-dimensional real manifold $\Gamma$, endowed with the Fubini-Study metric $g_{a b}$. The reason that $\Gamma$ plays the role of a quantum phase space is that Hamilton's equations on $\Gamma$ can be lifted to the Hilbert space to give the Schrödinger dynamics. This follows from the fact that $\Gamma$ has a natural symplectic structure, given by a nondegenerate antisymmetric tensor $\Omega_{a b}$, compatible with the metric $g_{a b}$ in the sense that $\nabla_{a} \Omega_{b c}=0$ and $\Omega_{a b} \Omega^{b c}=-\delta_{a}^{c}$, where $\Omega^{a b}=g^{a c} g^{b d} \Omega_{c d}$ and $\nabla_{a}$ denotes the standard covariant derivative, satisfying $\nabla_{a} g_{b c}=0$. Then the Schrödinger dynamics take the Hamiltonian form

$$
d x^{a}=2 \Omega^{a b} \nabla_{b} H d t
$$

where $d x^{a} / d t$ is tangent to the quantum phase space trajectory. The Hamiltonian function $H(x)$ on $\Gamma$ is given by the expectation of the energy operator at each point $x \in \Gamma$. More specifically, the expectation of the Hermitian operator $H_{\beta}^{\alpha}$ with respect to the Hilbert space vector $\psi^{\alpha}(\alpha, \beta=0,1, \cdots, n)$ is given by $H(x)=H_{\beta}^{\alpha} \bar{\psi}_{\alpha} \psi^{\beta} / \bar{\psi}_{\gamma} \psi^{\gamma}$. Since each point $x \in \Gamma$ corresponds to an equivalence class $\left\{\lambda \psi^{\alpha}, \lambda \in \mathbf{C}-0\right\}$ for some Hilbert space vector $\psi^{\alpha}$, it follows that $H(x)$ is defined globally on $\Gamma$. Conversely, one can show that such functions correspond to global solutions of the equation

$$
\nabla^{2} H=(n+1)(\bar{H}-H)
$$

where $\nabla^{2}$ is the Laplace-Beltrami operator on $\Gamma$. Here $\bar{H}=H_{\alpha}^{\alpha} /(n+1), H_{\alpha}^{\alpha}$ being the trace of the Hamiltonian. Thus $\bar{H}$ is the uniform average of the energy eigenvalues, corresponding to the thermal equilibrium energy of the system at high temperature. 


\section{STOCHASTIC DYNAMICS}

Now we generalise the Schrödinger dynamics by consideration of a diffusion process $x_{t}$ taking values in $\Gamma$. For this purpose we make use of standard techniques of stochastic differential geometry [4,5]. We shall examine the case of a Brownian motion with drift on $\Gamma$, determined by the covariant stochastic differential equation

$$
d x^{a}=\mu^{a} d t+\kappa \sigma_{i}^{a} d W_{t}^{i}
$$

where $\kappa$ is a constant, $\mu^{a}$ is a vector field, and the vectors $\sigma_{i}^{a}(i=1,2, \cdots, 2 n)$ constitute an orthonormal basis in the tangent space of $\Gamma$ such that $g^{a b}=\sigma_{i}^{a} \sigma_{j}^{b} \delta^{i j}$ and $\sigma_{i}^{a} \sigma_{j}^{b} g_{a b}=\delta_{i j}$. Here $d x^{a}$ is the covariant Ito differential [5], and the standard $2 n$-dimensional Wiener process $W_{t}^{i}$ satisfies $d W_{t}^{i} d W_{t}^{j}=\delta^{i j} d t$. In local coordinates $x^{\mathbf{a}}(\mathbf{a}=1,2, \cdots, 2 n)$, the Ito differential is given by

$$
d x^{a}=\delta_{\mathbf{a}}^{a}\left(d x^{\mathbf{a}}+\frac{1}{2} \kappa^{2} \Gamma_{\mathbf{b} \mathbf{c}}^{\mathbf{a}} g^{\mathbf{b c}} d t\right) .
$$

Here $\delta_{\mathbf{a}}^{a}$ is a coordinate basis for the given patch of $\Gamma$, and $\delta_{a}^{\mathbf{a}}$ the dual basis, such that for the covariant derivative $\nabla_{a} \xi^{b}$ of a vector field $\xi^{a}$ with components $\xi^{\mathbf{a}}=\delta_{a}^{\mathbf{a}} \xi^{a}$ we can write

$$
\delta_{\mathbf{b}}^{b} \delta_{a}^{\mathbf{a}}\left(\nabla_{b} \xi^{a}\right)=\frac{\partial \xi^{\mathbf{a}}}{\partial x^{\mathbf{b}}}+\Gamma_{\mathbf{b} \mathbf{c}}^{\mathbf{a}} \xi^{\mathbf{c}}
$$

The Ito differential (4) is constructed in such a way that the stochastic differential equation (3) is fully tensorial.

Suppose $\phi(x)$ is a smooth function on $\Gamma$, and define the process $\phi_{t}=\phi\left(x_{t}\right)$. It follows from Ito's lemma that $d \phi_{t}=\nabla_{a} \phi d x^{a}+\frac{1}{2} \nabla_{a} \nabla_{b} \phi d x^{a} d x^{b}$. Then the relation $d x^{a} d x^{b}=\kappa^{2} g^{a b} d t$ implies

$$
d \phi_{t}=\left(\mu^{a} \nabla_{a} \phi+\frac{1}{2} \kappa^{2} \nabla^{2} \phi\right) d t+\kappa \nabla_{a} \phi \sigma_{i}^{a} d W_{t}^{i}
$$

where $\nabla^{2} \phi=g^{a b} \nabla_{a} \nabla_{b} \phi$. Since the process $x_{t}$ is Markovian, there is a normalised density function $\rho(x, t)$ for the state at time $t$, characterised by a partial differential equation subject to specified initial conditions. The expectation of the process $\phi_{t}$ is thus

$$
E\left[\phi_{t}\right]=\int_{\Gamma} \phi(x) \rho(x, t) d V
$$

where $d V$ denotes the volume element on $\Gamma$. On the other hand, it follows from Ito's lemma (6) that

$$
\phi_{t}=\phi_{0}+\int_{0}^{t}\left(\mu^{a} \nabla_{a} \phi+\frac{1}{2} \kappa^{2} \nabla^{2} \phi\right) d s+\kappa \int_{0}^{t} \sigma_{i}^{a} \nabla_{a} \phi d W_{s}^{i}
$$

Here the integrands are valued at time $s$ at the point $x_{s} \in \Gamma$. Since the second integral above has vanishing expectation, we obtain

$$
E\left[\phi_{t}\right]=\phi_{0}+E\left[\int_{0}^{t}\left(\mu^{a} \nabla_{a} \phi+\frac{1}{2} \kappa^{2} \nabla^{2} \phi\right) d s\right] .
$$


Hence differentiating (7) and (9) with respect to $t$ and equating the results, we have

$$
\int_{\Gamma} \dot{\rho}(x, t) \phi(x) d V=\int_{\Gamma}\left(\mu^{a} \nabla_{a} \phi+\frac{\kappa^{2}}{2} \nabla^{2} \phi\right) \rho(x, t) d V,
$$

where $\dot{\rho}=\partial \rho / \partial t$. Integrating by parts, and using the fact that the resulting relation must hold for all $\phi(x)$, we find that $\rho(x, t)$ satisfies

$$
\frac{\partial}{\partial t} \rho(x, t)=-\nabla_{a}\left(\mu^{a} \rho\right)+\frac{1}{2} \kappa^{2} \nabla^{2} \rho .
$$

This is the covariant Fokker-Planck equation for the density function on $\Gamma$, corresponding to the stochastic process (3). The solution of (11) thus characterises the distribution of the diffusion (3) at time $t$, given an initial distribution $\rho(x, 0)$. In the case of a singular initial distribution, e.g., an initially pure state, we interpret $\rho(x, t)$ as a generalised function, i.e., it has to satisfy (10) for all smooth $\phi(x)$.

The foregoing results are valid on arbitrary compact Riemannian manifolds. We are concerned, however, with the case where $\Gamma$ is the quantum phase space $C P^{n}$, endowed with the Fubini-Study metric. Since $\Gamma$ is compact, equation (11) may admit nontrivial asymptotic $(t \rightarrow \infty)$ stationary solutions. We shall examine a simple situation in which this is the case, namely, when the drift is given by a gradient flow $\mu^{a}=-\frac{1}{2} \kappa^{2} \beta \nabla^{a} H$ generated by $H(x)$, where $\beta$ is a parameter. Then we can use a theorem of Zeeman [6] to show that there is a unique stationary solution for (11) of the form

$$
\rho(x)=\frac{\exp (-\beta H(x))}{Z(\beta)},
$$

where $Z(\beta)=\int_{\Gamma} \exp (-\beta H(x)) d V$. It follows that the probability density function $p(E)$ for the distribution of the energy function $H(x)$ is given by the formula $p(E)=$ $Z^{-1}(\beta) \Omega(E) \exp (-\beta E)$, where

$$
\Omega(E)=\int_{\Gamma} \delta(H(x)-E) d V
$$

is the density of states in $\Gamma$ for which $E \leq H(x)<E+d E$, and $Z(\beta)$ is the partition function. Another way of characterising the distribution (12) is that it maximises the entropy $S=-\int_{\Gamma} \rho(x) \ln \rho(x) d V$ for a given value of system energy $U=\int_{\Gamma} \rho(x) H(x) d V$. The theorem of Zeeman implies in the present context that, (12) is the asymptotic distribution of the process $x_{t}$ under essentially arbitrary initial conditions, and that $p(E)$ is the asymptotic energy distribution.

We have so far considered a process of the form (3) with the drift $\mu^{a}=-\frac{1}{2} \kappa^{2} \beta \nabla^{a} H$. This process, as such, does not yet make reference to the Schrödinger dynamics. If we view the drift term as a nonlinear correction to the underlying quantum evolution, we can represent the complete dynamics according to the prescription

$$
d x^{a}=\left(2 \Omega^{a b} \nabla_{b} H-\frac{1}{2} \kappa^{2} \beta \nabla^{a} H\right) d t+\kappa \sigma_{i}^{a} d W_{t}^{i},
$$

which in the limit $\kappa \rightarrow 0$ reduces to the ordinary Schrödinger dynamics. Due to the antisymmetry of $\Omega_{a b}$, inclusion of the symplectic term does not affect the resulting asymptotic 
state (12), since $\rho(x)$ is a function of $H(x)$. Hence, the analysis above shows that, given an arbitrary initial state (pure or general) on the quantum phase space, the dynamical law (14) necessarily takes that state into the thermal equilibrium state (12). In particular, the process (14) is ergodic on the energy surface, and asymptotically leads to a uniform distribution on each such surface. We note that (14) involves two parameters, $\beta$ and $\kappa$. The stationary solution for $\rho(x, t)$ depends only on $\beta$, which we identify as the inverse of the heat bath temperature. The parameter $\kappa$, which has the units $s^{-1 / 2}$, and may depend on $\beta$, determines the thermalisation time scale.

\section{HAMILTONIAN PROCESS}

Our next step is to study the stochastic process $H_{t}=H\left(x_{t}\right)$ associated with the Hamiltonian function. From Ito's lemma (6) for the process (14) we find

$$
d H_{t}=\frac{1}{2} \kappa^{2}\left(\nabla^{2} H_{t}-\beta V_{t}\right) d t+\kappa \nabla_{a} H \sigma_{i}^{a} d W_{t}^{i}
$$

Here $V_{t}=V\left(x_{t}\right)$, where $V(x)=g^{a b} \nabla_{a} H \nabla_{b} H$ is the squared energy uncertainty at the point $x \in \Gamma$, conditional on the pure state to which $x$ corresponds. Integrating (15) and taking its expectation, we have

$$
E\left[H_{t}\right]=H_{0}+\frac{1}{2} \kappa^{2} \int_{0}^{t} E\left[\nabla^{2} H_{s}-\beta V_{s}\right] d s .
$$

Let $U_{t}$ denote $E\left[H_{t}\right]$, the unconditional energy expectation, which can be interpreted as the total system energy at time $t$. Then by differentiating (16) we obtain

$$
\frac{\partial U_{t}}{\partial t}=\frac{1}{2} \kappa^{2}\left((n+1)\left(\bar{H}-U_{t}\right)-\beta E\left[V_{t}\right]\right)
$$

by use of the Laplace-Beltrami equation for $H_{t}$. This relation can be integrated to yield the solution for the time development of the system energy:

$$
\begin{aligned}
U_{t}= & \bar{H}+\left(H_{0}-\bar{H}\right) e^{-\frac{1}{2} \kappa^{2}(n+1) t} \\
& -\frac{1}{2} \kappa^{2} \beta \int_{0}^{t} \int_{\Gamma} e^{\frac{1}{2} \kappa^{2}(n+1)(s-t)} V(x) \rho(x, s) d V d s .
\end{aligned}
$$

In the limit $t \rightarrow \infty$ the only contribution is given by the trace term $\bar{H}$ and the integral of $V(x)$, and the resulting energy approaches the internal energy of the system in thermal equilibrium. In particular, in the high temperature limit $\beta \rightarrow 0$ the contribution from $V(x)$ vanishes, and we recover the uniform average of the energy eigenvalues. In the low temperature limit $\beta \rightarrow \infty$, the gradient term in the drift of (14) dominates, and the system is forced to fall to the ground state. It is interesting to observe, as a consequence of (17), that once thermal equilibrium is reached, we have the identity

$$
k T^{2} C=\operatorname{Var}[H]+(n+1) k T(U-\bar{H}),
$$

for the heat capacity $C=\partial U / \partial T$, where $\beta=1 / k T$ and $\operatorname{Var}[H]=(\Delta H)_{\rho}^{2}$ is the unconditional energy variance. This follows from the conditional variance formula 


$$
\operatorname{Var}[H]=E\left[\operatorname{Var}_{x}[H]\right]+\operatorname{Var}\left[E_{x}[H]\right]
$$

where $E_{x}[H]$ and $\operatorname{Var}_{x}[H]$ are, respectively, the conditional expectation and the conditional variance of the energy, when the system is in the pure state $x$. This relation expresses the total energy uncertainty by the sum of terms corresponding to quantum and thermal uncertainties.

As an illustration, consider the case of a spin one-half particle in heat bath. The state space is $C P^{1}$, which we view as a 2 -sphere, the north and the south poles corresponding to the upper and lower energy eigenstates, with energies $+h$ and $-h$, where $h$ is the magnetic moment of the particle times the external field strength. The symplectic flow gives rise to latitudinal circular orbits on the sphere, while the gradient flow is in the direction along the great circles passing through the two eigenstates, pushing the state towards the south pole. The equilibrium energy latitude is obtained by balancing the gradient flow and the Brownian fluctuations. If we let $\theta$ denote the angular coordinate for the state as measured from the north pole, then the squared energy uncertainty, given that the system is in a pure state at latitude $\theta$, is $\operatorname{Var}_{\theta}[H]=h^{2} \sin ^{2} \theta$. The conditional energy expectation is $E_{\theta}[H]=h \cos \theta$. Since $\bar{H}$ vanishes, the only contribution to the energy in (18) is from the volume integral, which gives $U=\beta^{-1}-h \operatorname{coth}(\beta h)$, in agreement with the result of a direct calculation of $E\left[E_{\theta}[H]\right]$. At infinite temperature the system energy corresponds to that of the equator, whereas for finite temperature the system energy corresponds to that of a latitude in the southern hemisphere. At zero temperature, the system collapses to the ground state.

\section{ENTROPY PRODUCTION}

Next we consider the dynamics of the total entropy of the system, given by $S_{t}=$ $-\int_{\Gamma} \rho(x, t) \ln \rho(x, t) d V$. Without loss of generality we can write

$$
\rho(x, t)=\frac{\exp (-\beta(H(x)+\eta(x, t)))}{\int_{\Gamma} \exp (-\beta(H(x)+\eta(x, t))) d V},
$$

for a general density function, where $\eta(x, t)$ determines the departure from thermal equilibrium. Substituting this expression into the formula for $S_{t}$, and making use of the FokkerPlanck equation (11) with drift as in (14), we find

$$
\frac{\partial S_{t}}{\partial t}=\frac{1}{2} \kappa^{2} \beta^{2} \int_{\Gamma}\left(\nabla^{a} \eta \nabla_{a} H+\nabla^{a} \eta \nabla_{a} \eta\right) \rho d V .
$$

On the other hand, for the total energy $U_{t}$ we have

$$
\frac{\partial U_{t}}{\partial t}=\frac{1}{2} \kappa^{2} \beta \int_{\Gamma}\left(\nabla^{a} \eta \nabla_{a} H\right) \rho d V
$$

Therefore, we obtain

$$
\frac{\partial S_{t}}{\partial t}=\beta \frac{\partial U_{t}}{\partial t}+\frac{1}{2} \kappa^{2} \beta^{2} \int_{\Gamma}\left(\nabla^{a} \eta \nabla_{a} \eta\right) \rho d V .
$$

This is the general formula for the entropy production associated with the process (14), which shows that 


$$
\frac{\partial S_{t}}{\partial t} \geq \beta \frac{\partial U_{t}}{\partial t}
$$

in all circumstances. In particular, if $U_{t}$ increases as a consequence of heating, then the entropy of the system also necessarily increases.

\section{EVOLUTION OF THE DENSITY MATRIX}

Finally we derive the dynamics of the density matrix $\rho_{\beta}^{\alpha}(t)$ associated with the process (14). Let us revert to homogeneous coordinates for the state space $C P^{n}$, and write $\Pi_{\beta}^{\alpha}(x)=$ $\psi^{\alpha}(x) \bar{\psi}_{\beta}(x) / \psi^{\gamma}(x) \bar{\psi}_{\gamma}(x)$ for the projection operator onto the pure state $x \in \Gamma$ represented by the Hilbert space vector $\psi^{\alpha}(x)$. From equations (7) and (10) it follows that for a general (possibly nonlinear) observable $\phi(x)$ on $\Gamma$ we have

$$
\begin{aligned}
\frac{\partial}{\partial t} E\left[\phi\left(x_{t}\right)\right]= & \int_{\Gamma}\left(2 \Omega^{a b} \nabla_{a} \phi \nabla_{b} H+\frac{1}{2} \kappa^{2} \nabla^{2} \phi\right. \\
& \left.-\frac{1}{2} \kappa^{2} \beta g^{a b} \nabla_{a} \phi \nabla_{b} H\right) \rho(x, t) d V .
\end{aligned}
$$

However, if $\phi(x)$ is given, more specifically, by the conditional expectation of an ordinary linear quantum mechanical observable $\phi_{\beta}^{\alpha}$, then $\phi(x)=\phi_{\alpha}^{\beta} \Pi_{\beta}^{\alpha}(x)$. Thus for a linear observable we have $E\left[\phi\left(x_{t}\right)\right]=\phi_{\alpha}^{\beta} \rho_{\beta}^{\alpha}(t)$, where

$$
\rho_{\beta}^{\alpha}(t)=\int_{\Gamma} \Pi_{\beta}^{\alpha}(x) \rho(x, t) d V
$$

is the time dependent density matrix associated with the state $\rho(x, t)$. To obtain the equation of motion for $\rho_{\beta}^{\alpha}(t)$ we need to evaluate the three terms in the integrand on the right of (26). These are given as follows. For the commutator term we have

$$
\Omega^{a b} \nabla_{a} \phi \nabla_{b} H=\frac{1}{2} i\left(\phi_{\gamma}^{\beta} H_{\alpha}^{\gamma}-H_{\gamma}^{\beta} \phi_{\alpha}^{\gamma}\right)
$$

for the term involving the Laplace-Beltrami operator we find

$$
\nabla^{2} \phi=\left(\phi_{\gamma}^{\gamma} \delta_{\alpha}^{\beta}-(n+1) \phi_{\alpha}^{\beta}\right) \Pi_{\beta}^{\alpha}
$$

and for the anticommutator term we obtain

$$
g^{a b} \nabla_{a} \phi \nabla_{b} H=\frac{1}{2}\left(\phi_{\gamma}^{\beta} H_{\alpha}^{\gamma}+H_{\gamma}^{\beta} \phi_{\alpha}^{\gamma}\right) \Pi_{\beta}^{\alpha}-\left(\phi_{\alpha}^{\beta} H_{\gamma}^{\delta}\right) \Pi_{\beta}^{\alpha} \Pi_{\delta}^{\gamma} .
$$

Inserting these expressions into (26), and noting that the result must hold for arbitrary $\phi_{\alpha}^{\beta}$, we deduce that

$$
\begin{aligned}
\frac{\partial}{\partial t} \rho_{\beta}^{\alpha}= & i\left[H_{\gamma}^{\alpha} \rho_{\beta}^{\gamma}-\rho_{\gamma}^{\alpha} H_{\beta}^{\gamma}\right]-\frac{1}{4} \kappa^{2} \beta\left(H_{\gamma}^{\alpha} \rho_{\beta}^{\gamma}+\rho_{\gamma}^{\alpha} H_{\beta}^{\gamma}\right) \\
& +\frac{1}{2} \kappa^{2}\left(\delta_{\beta}^{\alpha}-(n+1) \rho_{\beta}^{\alpha}+\beta H_{\gamma}^{\delta} \rho_{\beta \delta}^{\alpha \gamma}\right)
\end{aligned}
$$


Here the density matrix $\rho_{\beta}^{\alpha}(t)$ is given by (27), the first moment of the projection operator $\Pi_{\beta}^{\alpha}(x)$, whereas $\rho_{\alpha \gamma}^{\beta \delta}(t)$ is the second moment of $\Pi_{\beta}^{\alpha}(x)$, given by

$$
\rho_{\beta \delta}^{\alpha \gamma}(t)=\int_{\Gamma} \Pi_{\beta}^{\alpha}(x) \Pi_{\delta}^{\gamma}(x) \rho(x, t) d V
$$

Equation (31) is the dynamical law for the density operator, which takes an arbitrary initial state into an equilibrium state, with heat bath temperature $T$. The first term on the right of (31) leads to the Liouville equation of linear quantum dynamics, while the general nonequilibrium process has a richer structure. The emergence of the second moment term in (31) can also be interpreted by analogy with the renormalisation group equations.

\section{DISCUSSION}

The model we have described here is surprisingly tractable, and has many attractive features. Experimental support could be pursued in two stages. First, we point out that it is not difficult, in the case of the canonical $\Gamma$-ensemble, to derive explicit formulae for the partition function $Z(\beta)$, the state density $\Omega(E)$, the density matrix $\rho_{\beta}^{\alpha}$, and the second

moment $\rho_{\beta \delta}^{\alpha \gamma}$. If these results turn out to give a better account of equilibrium phenomena than the conventional approach in some context, then the next step would be to look for effects resulting from the higher moment term in the nonequilibrium dynamical law (31).

DCB is grateful to PPARC for financial support.

* Electronic address: d.brody@damtp.cam.ac.uk

† Electronic address: lane@ml.com

1. L. E. Reichl, A Modern Course in Statistical Physics (Edward Arnold, London 1980); R. Kubo, M. Toda, N. Hashizume, and N. Saito, Statistical Physics I, II (SpringerVerlag, Berlin 1983, 1985).

2. T. W. B. Kibble, Commun. Math. Phys. 65, 189 (1979); G. W. Gibbons, J. Geom. Phys. 8, 147 (1992); L. P. Hughston, in Twistor Theory, edited by S. Huggett (Marcel Dekker, New York, 1995); D. C. Brody and L. P. Hughston, Phys. Rev. Lett. 77, 2581 (1996); Proc. Roy. Soc. London 454, 2445 (1998); A. Ashtekar and T. A. Schilling, in On Einstein's Path, edited by A. Harvey (Springer-Verlag, Berlin 1998).

3. S. Weinberg, Phys. Rev. Lett. 62, 485 (1989).

4. N. Ikeda and S. Watanabe, Stochastic Differential Equations and Diffusion Process (North-Holland, Amsterdam 1989); M. Emery, Stochastic Calculus on Manifolds (Springer-Verlag, Berlin 1989).

5. L. P. Hughston, Proc. Roy. Soc. London 452, 953 (1996).

6. E. C. Zeeman, Nonlinearity, 1, 115 (1988). 